\title{
Metzincin's canonical methionine is responsible for the structural integrity of the zinc-binding site
}

\author{
Anselm E. Oberholzera, Mario Bumann ${ }^{a, b}$, \\ Thomas Hege, Santina Russo ${ }^{\circ}$ and \\ Ulrich Baumann*
}

Department of Chemistry and Biochemistry, University of Bern, CH-3012 Bern, Switzerland

${ }^{*}$ Corresponding author

e-mail: ulrich.baumann@ibc.unibe.ch

\begin{abstract}
The metzincins constitute a subclan of metalloproteases possessing a HEXXHXXGXXH/D zinc-binding consensus sequence where the three histidines are zinc ligands and the glutamic acid is the catalytic base. A completely conserved methionine is located downstream of this motif. Families of the metzincin clan comprise, besides others, astacins, adamalysins proteases, matrix metalloproteases, and serralysins. The latter are extracellular $50 \mathrm{kDa}$ proteases secreted by Gram-negative bacteria via a type I secretion system. While there is a large body of structural and biochemical information available, the function of the conserved methionine has not been convincingly clarified yet. Here, we present the crystal structures of a number of mutants of the serralysin member protease $\mathrm{C}$ with the conserved methionine being replaced by Ile, Ala, and His. Together with our former report on the leucine and cysteine mutants, we demonstrate here that replacement of the methionine side chain results in an increasing distortion of the zinc-binding geometry, especially pronounced in the $\chi^{2}$ angles of the first and third histidine of the consensus sequence. This is correlated with an increasing loss of proteolytic activity and a sharp increase of flexibility of large segments of the polypeptide chain.
\end{abstract}

Keywords: crystal structure; directed mutagenesis; metalloprotease; metzincin; serralysin.

\section{Introduction}

Zinc-dependent endo-metalloproteases occur in all kingdoms of life and are involved in manifold biological functions. Many of them are characterized by a consensus sequence HEXXH, where the histidines are zinc ligands and the glutamic acid functions as catalytic base. A third zinc ligand is provided by an amino acid side-chain (His, Glu, Asp) downstream of this motif and the coordination

\footnotetext{
a These authors contributed equally to this work.

bPresent address: MRC France, CRG BM14 ESRF, B.P. 220, F-38043 Grenoble Cedex, France.

cPresent address: Swiss Light Source, Paul Scherrer Institute, $\mathrm{CH}-5232$ Villigen, Switzerland.
}

sphere is completed by the activated water molecule that attacks the peptide bond in the course of the reaction (Jiang and Bond, 1992; Tronrud et al., 1992). These enzymes are grouped in clan MA of the MEROPS protease database (Rawlings et al., 2006). The metzincins are a subclan, possessing an elongated HEXXH$X X G X X(H / D)$ zinc-binding motif with the last amino acid (mostly His) being the third zinc ligand (Bode et al., 1993; Stocker et al., 1995; Gomis-Ruth, 2003, 2009). The name of this subclan originates from a conserved methionine located in a 1,4-turn (so-called 'Met-turn'), which is subjacent to the base of the pyramidal arrangement of the three zinc ligands (Bode et al., 1993). Protease families belonging to the metzincins are, besides others, the astacins (Bond and Beynon, 1995), matrix metalloproteases (MMPs; Nagase et al., 2006), adamalysins (Blobel, 2005), and serralysins (Maeda and Morihara, 1995). The latter are proteases secreted by Gram-negative bacteria utilizing a type I secretion system. Typical members are serralysin from Serratia marcescens, alkaline protease from Pseudomonas aeruginosa and protease C (PrtC) from Erwinia chrysanthemi. These $50 \mathrm{kDa}$ proteins possess, besides an N-terminal approximately 220 amino acid long protease domain, a C-terminal $\beta$-roll moiety which is formed by tandem repeats of the glycine-rich nonapeptide GGXGXDXLX. This domain binds calcium and probably functions as an intramolecular chaperone (Baumann et al., 1993; Meier et al., 2007) (Figure 1A). Serralysins are classical metzincins where His-188, His-192, and His-198 are the zinc ligands and the Metturn possesses the sequence 224-SIMSY-228. The tyrosine (Tyr-228) in this element stabilizes the tetrahedral transition state of the hydrolysis reaction (Hege and Baumann, $2001 b)$ and is not present in all metzincin families (Figure $1 \mathrm{~B})$. Another unique feature of the serralysins is the loop comprising residues 201-210 in PrtC (drawn in orange in Figure 1), which is quite flexible in the apo-state and undergoes an induced-fit closure upon substrate binding.

The various metzincin families are rather distant to each other in evolution, exhibiting sequence identities of typically much less than $20 \%$, and sequence alignments frequently pick up only the zinc-binding consensus motif. Between these families, the Met-turn is located in a nonconserved sequence stretch and the spacing between zinc-binding consensus motif and Met-turn varies. Nevertheless, as identified by structural alignment, the methionine always occupies the same spatial position and is in the same conformation (Figure 1). It lies below the base of the pyramid formed by the three histidines which are the amino acid zinc ligands. The sulfur atom is approximately $6 \AA$ away from the metal ion and its lone pairs point in the opposite direction. The carbonyl oxygen accepts a hydrogen bond from the $\mathrm{N} \delta 1$ of His-188, which is the first zinc ligand. 

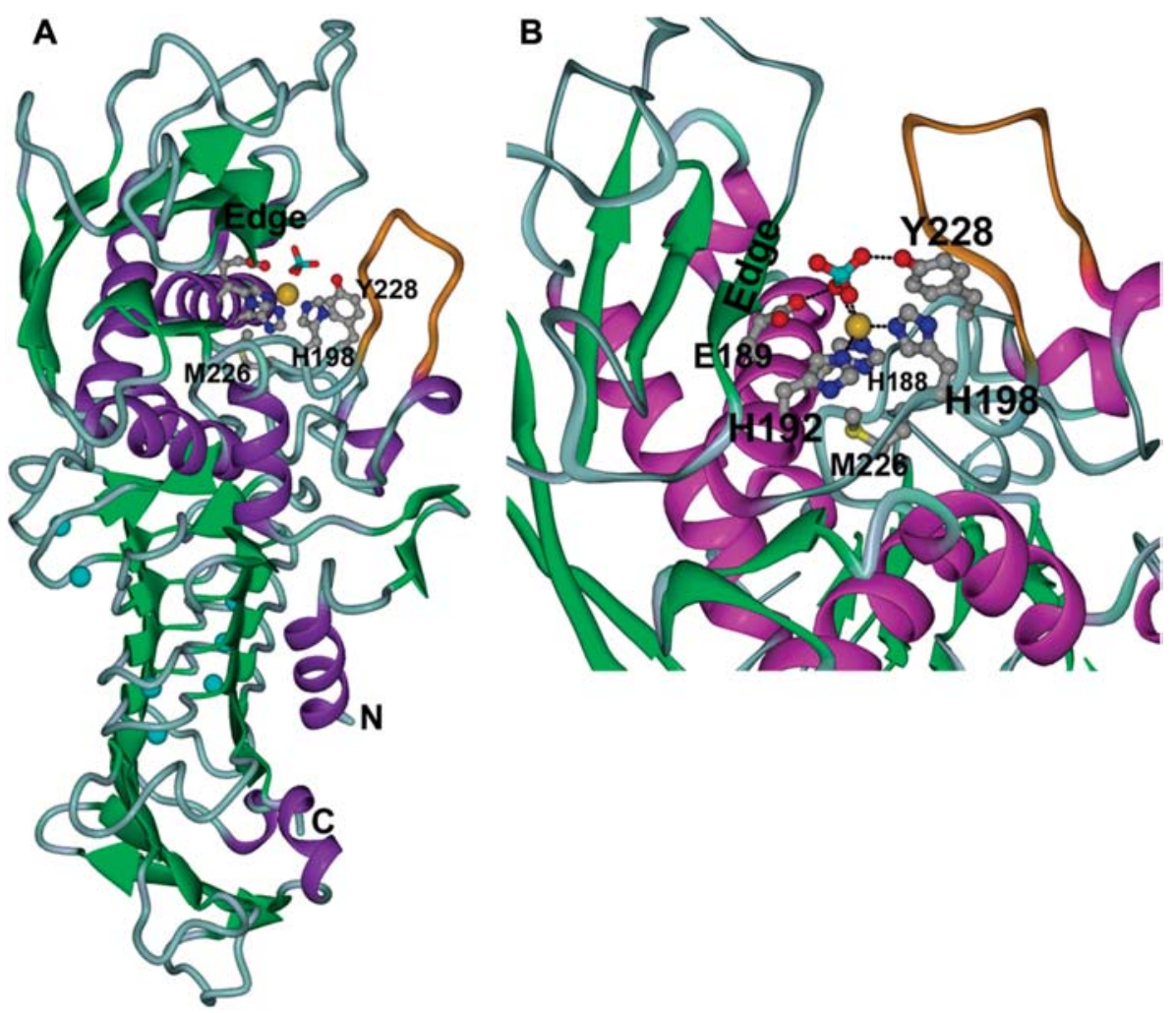

Figure 1 Overall structure and active center of wild-type PrtC.

(A) Ribbon diagram. The proteolytic domain is at the top, the $\beta$-roll domain at the bottom. Zinc is shown as golden sphere, calcium ions in light blue. A phosphate ion from the crystallization buffer coordinating to the zinc is shown as cyan and red sticks. The flexible loop (residues 200:206) is shown in orange. (B) Close-up of the active site. The substrate-binding edge strand is labeled as 'edge'. The sulfur atom of the methionine is depicted in yellow.

Methionine is not a conservative amino acid and ranks among the top $25 \%$ most rapidly mutating amino acids, being replaced most frequently by leucine, isoleucine, or valine (Jones et al., 1992; Tourasse and Li, 2000). Hence, owing to the apparently perfect conservation in a large set of very distantly related proteins, one should expect that the Met-turn methionine is very important for proteolytic activity and/or structural integrity. However, experimental studies replacing this residue by other amino acids in different metzincins did not deliver unequivocal results. In the case of MMPs, substitution of Met by selenomethionine (Qoronfleh et al., 1995; Pieper et al., 1997) or Leu or Ser (Butler et al., 2004) showed none or only minor changes in catalytic activity. Similar results were obtained by introduction of difluoromethionine in the serralysin alkaline protease (Walasek and Honek, 2005). On the other hand, mutation to Leu, lle, or Ser in tumor necrosis factor $\alpha$-converting enzyme led to a loss of ectodomain shedding (Perez et al., 2007). Our previous mutagenesis study of PrtC showed decreased proteolytic activity of mutants bearing Leu, Ile, and Ala at the position of Met-226. Crystal structures of the leucine mutant M226L and a double mutant EM189,226KC revealed subtle changes in the active site (Hege and Baumann, 2001a). The latter construct had an unwanted additional mutation of the active site glutamic acid, thus inactivating the enzyme completely. This allowed for more convenient purification and crystallization procedures.

In order to put the effects of methionine mutations on a more extended structural basis, we prepared more mutants and characterized their proteolytic activity. Fur- thermore, we determined the crystal structures of double mutants carrying Ile, Ala, and His at the methionine position and report the results here.

\section{Results}

\section{Proteolytic activity}

In the past, we have reported Michaelis-Menten parameters for the M226L, M226l, and M226A mutants (Hege and Baumann, 2001a). Other mutants, such as $\mathrm{M} 226 \mathrm{H}$ and $\mathrm{M} 226 \mathrm{~N}$, could not be purified. Since the proteins were secreted into the extracellular medium and no other proteases were present there, we assayed proteolytic activities directly from the supernatant employing a resorufin-casein assay, normalized for protein concentrations determined by a BioRad assay as reported previously (Hege and Baumann, 2001b; see Figure 2). The results are in qualitative agreement with our previous data. Not surprisingly, the specific activity decreases Met $>$ Leu$>\mathrm{lle}>\mathrm{His}>\mathrm{Ala}$, with the conservative mutations Leu and lle having some $85 \%$ and $50 \%$ of the wild-type activity, respectively. The levels of secreted protein decrease in the same order, indicating some defect in synthesis and secretion or stability of the mutants.

\section{Crystal structure analysis}

Since yield and purity of some of the single methionine mutants was not sufficient for structural studies, we 


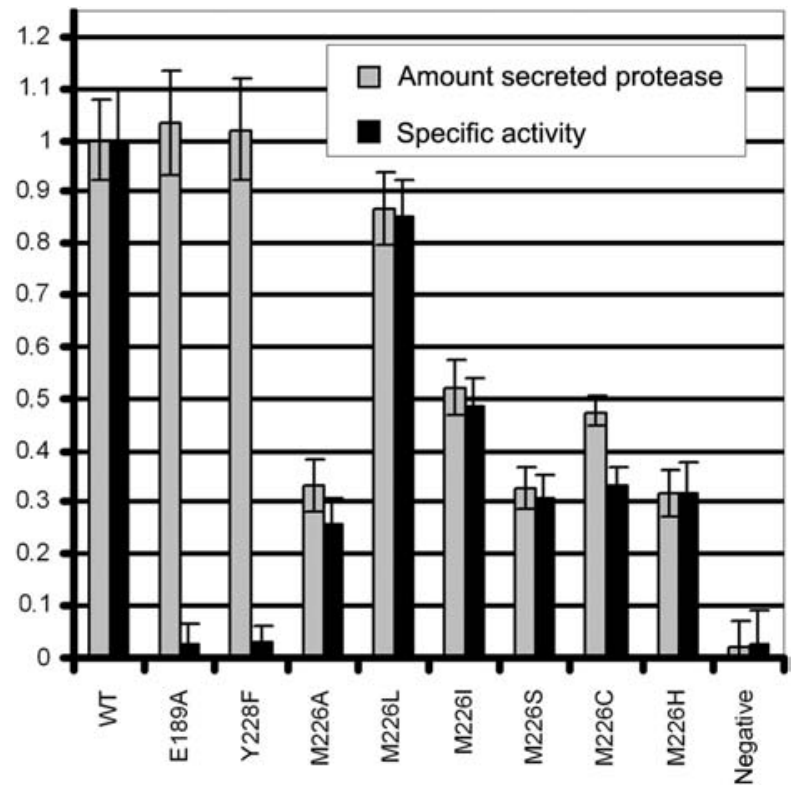

Figure 2 Activity assays.

Activity was assayed using resorufin-casein and media supernatant after spinning down the cells as described in the materials and methods section. Shown is the average of three independent measurements. Light bars indicate the ratio of secreted protein to cell density, and the dark bars indicate the specific activity. Values for the wild-type protein were normalized to 1.0. Cells carrying only the transport machinery plasmid were used as negative control ('negative').

introduced an additional mutation E189A, knocking out the catalytic base and thus inactivating the mutants completely. This greatly helped in purification and crys- tallization. Relevant statistics are given in Table 1. No significant changes in the active site were introduced by this mutation (data not shown), besides the disappearance of a zinc-bound phosphate ion originating from the crystallization buffer and a slight movement of His-198. Therefore, we conclude that the differences we observe for the Met-226 mutants originate largely from the replaced methionine (Qoronfleh et al., 1995; Pieper et al., 1997; Walasek and Honek, 2005).

An overlay of the active sites of the more conservative mutants (M226L, M226I, M226C) shows mainly a change in the geometry of the imidazole side chains of the coordinating histidine residues (Figure 3 ). In particular, the $\chi^{1}$ angle of His-188 increases with decreasing activity (Table 2) and with decreasing size of the side-chain at position 226. This change in side-chain conformation causes a movement of the imidazole ring towards the cavity created by the mutation. The effect is most dramatic by cutting down the methionine side-chain to the $\mathrm{C}^{\beta}$ atom, i.e., in the M226A mutant (Figure 4). As can be seen in Figure $4 \mathrm{~A}$, the zinc, which is clearly present as documented by a large peak in the anomalous difference Fourier map, moves downwards by approximately $3 \AA$, while the imidazole side-chain of His-188 is nearly perpendicular to the orientation found in the wild-type enzyme. In order to keep the coordination sphere, His-198 changes position as well. This latter change is possible due to a dramatic order-disorder transition: amino acids 18-61 and 199-238, all very well defined in all the other mutants with the exception of $\mathrm{M} 226 \mathrm{H}$, become completely disordered. The second segment comprises the Met-turn and would clash with the new side-chain conformation of His-198. In other words, the polypeptide chain cov-

Table 1 Data collection and refinement.

\begin{tabular}{|c|c|c|c|c|c|}
\hline Protein & M226I-E189A & $\begin{array}{l}\text { M226A- } \\
\text { E189A_inh }\end{array}$ & $\begin{array}{l}\text { M226A- } \\
\text { E189A_SLS }\end{array}$ & $\begin{array}{c}\text { M226A- } \\
\text { E189A_DESY }\end{array}$ & $\begin{array}{c}\text { M226H- } \\
\text { E189A }\end{array}$ \\
\hline X-ray source & DESY X11 & Ru300 & SLS PXI & DESY X11 & DESY X11 \\
\hline Unit cell a $(\AA)^{a}$ & 102.41 & 101.96 & 102.26 & 101.89 & 102.11 \\
\hline Unit cell c ( $(\AA)$ & 121.03 & 123.22 & 123.40 & 122.52 & 122.86 \\
\hline Wavelength $(\AA ̊)$ & 0.812 & 1.542 & 1.281 & 0.812 & 0.812 \\
\hline $\begin{array}{l}\text { Resolution }(\AA) \\
\text { (outer shell) }\end{array}$ & $\begin{array}{l}50.0-1.75 \\
(1.78-1.75)\end{array}$ & $\begin{array}{l}50.0-1.95 \\
(1.98-1.95)\end{array}$ & $\begin{array}{l}26.6-2.05 \\
(2.16-2.05)\end{array}$ & $\begin{array}{l}50.0-2.13 \\
(2.17-2.13)\end{array}$ & $\begin{array}{c}50.0-1.77 \\
(1.80-1.77)\end{array}$ \\
\hline $\mathrm{R}_{\text {merge }}(\%)$ & $4.8(32.9)$ & $3.4(18.4)$ & $11.7(19.1)$ & $8.4(39.7)$ & $4.0(17.4)$ \\
\hline Completeness (\%) & $99.9(98.5)$ & $99.6(100.0)$ & $99.9(99.8)$ & $99.9(100.0)$ & $100.0(100)$. \\
\hline Redundancy & $5.6(3.8)$ & $6.0(5.1)$ & $7.5(5.8)$ & $6.7(3.8)$ & $7.4(5.7)$ \\
\hline $\begin{array}{l}\text { I/sigma }(I) \\
\text { refinement }\end{array}$ & $23.0(5.0)$ & $28.3(7.5)$ & $15.5(6.1)$ & 12.9 (3.3) & $45.1(10.2)$ \\
\hline $\begin{array}{l}\text { No. of reflections } \\
\text { work (test) set }\end{array}$ & 74320 (2183) & 54271 (1631) & 47147 (1412) & 41498 (1260) & 72453 (2138) \\
\hline No. atoms protein & 3525 & 2937 & 2961 & 2947 & 3554 \\
\hline $\mathrm{Ca}^{2+} / \mathrm{Zn}^{2+}$ & $7 / 1$ & $7 / 1$ & $7 / 1$ & $7 / 0$ & $7 / 2^{c}$ \\
\hline Water & 548 & 512 & 481 & 227 & 486 \\
\hline $\mathrm{R} / \mathrm{R}_{\text {free }}(\%)$ & $15.5 / 17.9$ & $15.3 / 16.6$ & $14.8 / 17.0$ & $18.2 / 20.7$ & $16.6 / 18.7$ \\
\hline RMS bonds $(\AA \AA)$ & 0.017 & 0.007 & 0.007 & 0.005 & 0.009 \\
\hline RMS angles (deg.) & 1.56 & 1.0 & 0.97 & 0.88 & 1.63 \\
\hline $\begin{array}{l}\text { Ramachandran } \\
\text { outliers }(\%)^{\mathrm{b}}\end{array}$ & 0 & 0 & 0 & 0 & 0 \\
\hline
\end{tabular}

aCrystals belong to space group $\mathrm{P}_{1} 21$.

bAccording to Lovell et al. (2003) and Richardson (2003).

cThe two $\mathrm{Zn}^{2+}$ ions occupy alternating sites with approximately $50 \%$ occupancy.

The numbers in parentheses refer to the outer resolution shell. 
ering the left side of the active center in Figure 4B-D (right side in Figure 1) becomes completely disordered. A patch of peptide-shaped electron density is clearly visible in the active site, forming an antiparallel $\beta$-strand with the edge of the sheet in the protease domain. It is unlikely that this peptide is one of the proteolysis products sometimes observed in the active centers of serralysins (Baumann et al., 1993): firstly, the E189A mutation inactivates PrtC completely, and secondly the putative peptide extends over the zinc-binding position, which means it resembles more the educt than the products of the hydrolytic reaction.

However, the situation in the EM189,226AA mutant is even more complicated, owing to the additionally occurring complete loss of the zinc ion, as revealed by analysis of other crystals. Two out of four datasets show the condition depicted in Figure 4, while the remaining two crystals exhibit an entire loss of the zinc ion concomitant with a complete rearrangement of the active-site histidines (Figure 5, Table 2).

Another variation of the distortion of the active site could be observed in the EM189,226AH mutant. Here, an alternative zinc-binding site with approximately 50\% occupancy, as derived by comparison of B-factors from neighboring atoms and the situation in other mutants, is created by the presence of the additional imidazole group, as is clearly revealed by the imaginary electron density component (Figure 6). His-188 occurs in two alternative conformations coordinating to both zinc sites. Polypeptide segments 18-61 and 199-238 exhibit slightly higher average B-factors than in the E189A mutant, but are clearly visible with the exception of residues 203-210 belonging to the active center loop.

\section{Discussion}

Currently, some 800 members of the metzincin clan are known (Gomis-Ruth, 2003, 2009). All of them appear to possess the conserved methionine, although this residue is sometimes only tentatively identified by sequence alignments. Mutagenesis of such a conserved amino acid would be expected to exhibit large effects on activity and stability. However, conservative substitution by Leu or lle, the naturally most frequently occurring mutations, displays only moderate effects. However, the previously reported distortions of the zinc environment have been confirmed, and they become increasingly larger with decreasing side-chain size at position 226. In order to assess the fit of the methionine side-chain compared to other amino acids, we calculated the shape complementarity value (SC value) (Lawrence and Colman, 1993) from our X-ray structures. A perfect fit would be indicated by a value of 1.0. The values for wild-type, M226L, M226I, and $\mathrm{M} 226 \mathrm{C}$ mutants are $0.81,0.73,0.56$, and 0.42 , respectively. This clearly shows that in the jigsaw packing of hydrophobic amino acids at the base of the zincbinding pyramid, methionine is best fitting. Thus, even replacement by leucine leads to observable distortions and a reduction in activity by approximately $15 \%$. While this effect is small, it might nevertheless contribute to the selection pressure on the methionine. It should be noted

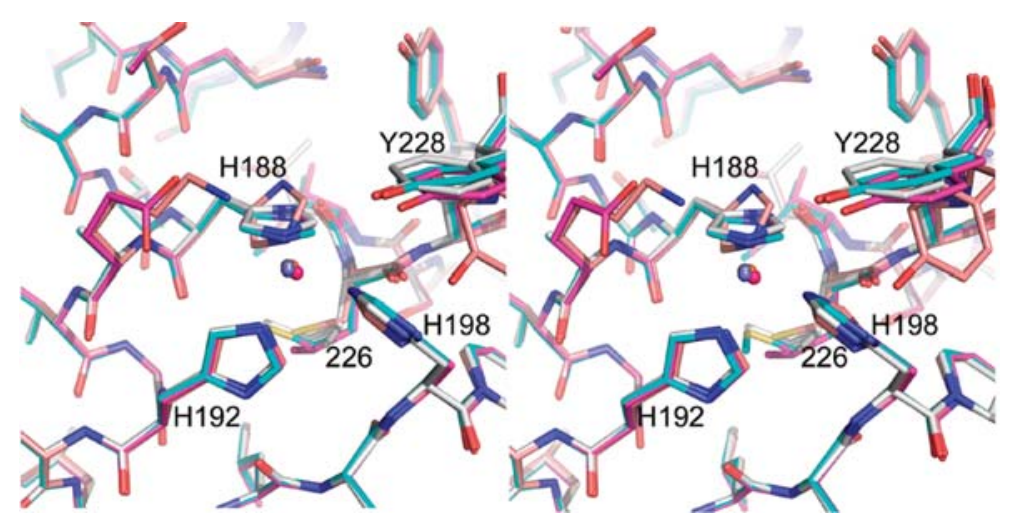

Figure 3 Active centers of M226 mutants revealing a distortion of the $\mathrm{Zn}^{2+}$-binding site and a shift in the coordinating histidines. Stereo view of mutants E189A (cyan carbon atoms), M226L (magenta), EM189,226Al (gray), and EM189,226KC (wheat). The phosphate ion still present in the $\mathrm{M} 226 \mathrm{~L}$ structure has been omitted as well as the zinc-coordinating water molecules. The systematic change in the side-chain dihedrals, especially $\chi^{2}$ of His-188, with decreasing size of the side-chain at position 226 is clearly visible (compare to Table 2).

Table 2 Side-chain dihedral angles of active-site histidines.

\begin{tabular}{|c|c|c|c|c|c|c|c|c|}
\hline & $\begin{array}{l}\text { Wild- } \\
\text { type }\end{array}$ & E189A & $\begin{array}{l}\text { M226L- } \\
\text { E189A }\end{array}$ & $\begin{array}{l}\text { M226I- } \\
\text { E189A }\end{array}$ & $\begin{array}{l}\text { M226C- } \\
\text { E189K }\end{array}$ & $\begin{array}{l}\text { M226H- } \\
\text { E189A }\end{array}$ & $\begin{array}{c}\text { M226A- } \\
\text { E189A_inh }\end{array}$ & $\begin{array}{c}\text { M226A- } \\
\text { E189A_DESY }\end{array}$ \\
\hline $\mathrm{H} 188 \chi^{1}$ & 176 & 176 & 177 & 177 & 170 & 174 & -169 & -71 \\
\hline $\mathrm{H} 188 \chi^{2}$ & 103 & 104 & 109 & 111 & 172 & 140 & 171 & 175 \\
\hline $\mathrm{H} 192 \chi^{1}$ & -73 & -72 & -68 & -67 & -67 & -79 & -71 & -172 \\
\hline $\mathrm{H} 192 \chi^{2}$ & -44 & -43 & -44 & -41 & -38 & -34 & -41 & 104 \\
\hline $\mathrm{H} 198 \chi^{1}$ & -60 & -68 & -59 & -61 & -60 & -69 & -49 & -49 \\
\hline $\mathrm{H} 198 \chi^{2}$ & 145 & 152 & 143 & 143 & 125 & 126 & 111 & 112 \\
\hline
\end{tabular}


A
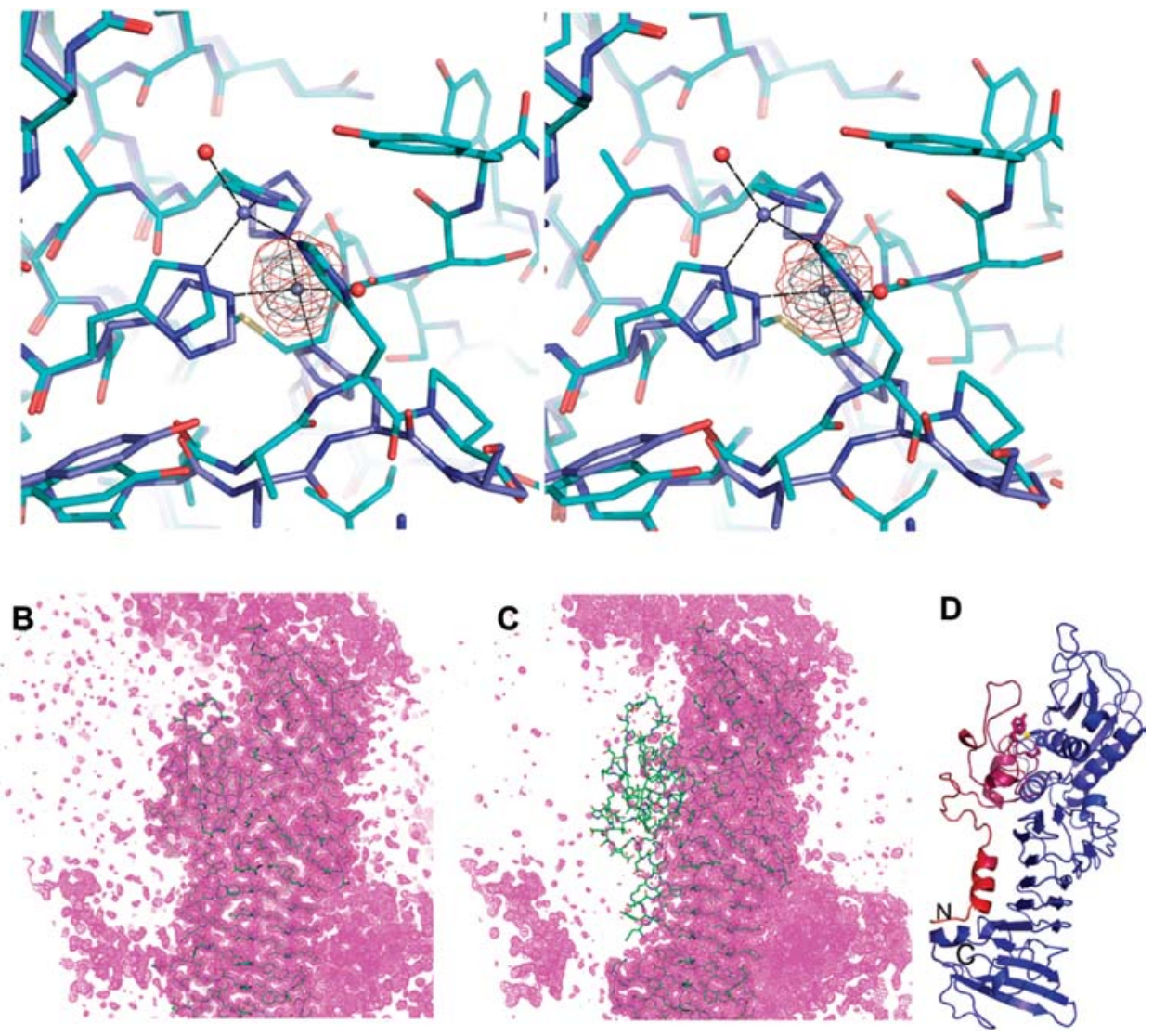

Figure 4 The EM189,226AA mutant as seen with the in-house and SLS data.

(A) Stereo view of an overlay E189A (cyan carbons, zinc shown as blue sphere) and EM189,226AA (blue carbon atoms, zinc depicted as gray sphere). Coordinating water molecules are shown as red spheres. An imaginary Fourier map is contoured at 10 and 15 standard deviations above the mean (magenta and gray mesh, respectively; data collected at SLS). (B) $2 \mathrm{~F}_{\mathrm{o}}-\mathrm{F}_{\mathrm{c}}$ electron density (contour level 1 sigma) of E189A. (C) $2 \mathrm{~F}_{0}-\mathrm{F}_{\mathrm{c}}$ density map contoured at 1.0 sigma for the EM189,226AA double mutant. The orientation is similar as in panel (B). (D) Ribbon diagram of EM189,226AA colored according to B-factors from blue to red $\left(<25 \AA^{2}\right.$ : blue; $>220 \AA^{2}$ : red). The orientation is approximately 180 degrees around the vertical from Figure 1 and similar as in panels (B) and (C). M226 and Y228 are shown as sticks as well as $\mathrm{H} 188$ and H192. The purple and red segments are completely invisible and have been omitted in panel $(A)$.

that most other studies have replaced the methionine with selenomethionine or difluoromethionine, i.e., amino acids with different electronic properties at the $\delta$-position of the side-chain but with a very similar size and shape
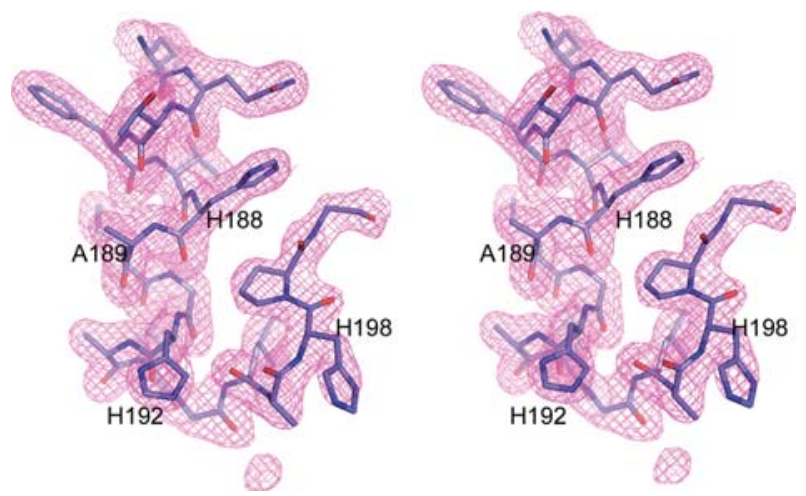

Figure 5 Active site of EM189,226AA as seen in data from DESY.

The $2 \mathrm{~F}_{\mathrm{o}}-\mathrm{F}_{\mathrm{c}}$ map (magenta) is contoured at 1.0 sigma above the mean. Anomalous difference Fourier maps show no signal for a $\mathrm{Zn}^{2+}$ ion anywhere close to the active site. This is in contrast to Figure $4 \mathrm{~A}$ where the $\mathrm{Zn}^{2+}$ site is shifted.

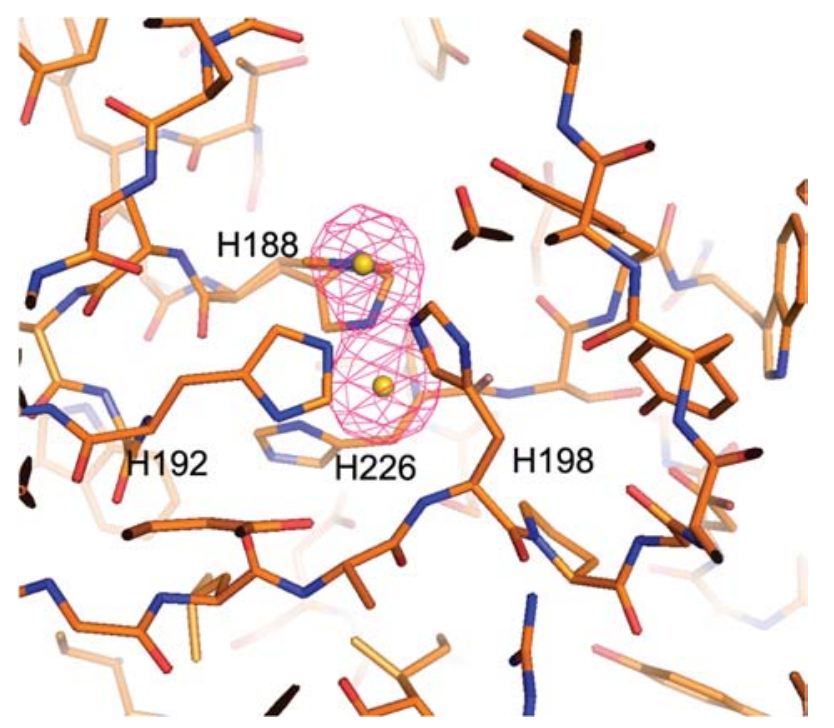

Figure 6 The EM189, 226AH mutant possesses two alternative $\mathrm{Zn}^{2+}$ sites.

The imaginary component of the electron density is shown in pink and contoured at 8 standard deviations above the mean (data collected at DESY). Zinc ions are shown as yellow spheres. 
(Qoronfleh et al., 1995; Pieper et al., 1997; Walasek and Honek, 2005). Only the substitution of methionine by serine in MMP-2 (Butler et al., 2004) does not fit into our observations on PrtC. While in MMP2 no difference in activity and stability has been detected, in PrtC the M226S mutant exhibits approximately $30 \%$ of the wildtype activity, very similar to the cysteine mutant. Further studies will be needed to resolve these conflicting results and to attain a deeper understanding of the conservation of the methionine.

\section{Materials and methods}

\section{Activity assay}

LB medium ( $5 \mathrm{ml}: 50 \mu \mathrm{g} / \mathrm{ml}$ kanamycin, $50 \mu \mathrm{g} / \mathrm{ml}$ chloramphenicol) was inoculated with a single colony carrying two plasmids pRUW520 (PrtC) and pRUW4inh1 (transport machinery) and incubated under shaking at $37^{\circ} \mathrm{C}$ overnight. The cell density was measured at $500 \mathrm{~nm}$. Cells were removed by centrifugation and the protein concentration in the supernatant was measured by a Bradford assay. An aliquot was precipitated by trichloroacetic acid and analyzed on sodium dodecyl sulfate polyacrylamide gel electrophoresis. Proteolytic activity was assayed employing resorufin-casein (Roche Diagnostics, Basel, Switzerland) according to the manufacturer's instructions. Cells transformed only with pRUW4inh1 and grown in LB medium without kanamycin served as negative control.

\section{Mutagenesis, protein expression, and purification}

Site-directed mutagenesis was performed as described previously (Jones and Winistorfer, 1992; Hege and Baumann, 2001b) using the E189A DNA as template. Oligonucleotides were ordered from MWG Biotec (Ebersberg, Germany). The helper primer had the sequence $5^{\prime}$-CGC GGC CTC GAG CAA GAC GTT TCC CG-3'. The following mutagenic primers were employed (base changes are underlined):

M226l sense: 5'-CAA TTC AGT ATC ATA AGC TAC TGG GG-3'; M226A sense: 5'-CAA TTC AGT ATC GCG AGC TAC TGG GG-3'; and

M226H sense: 5'-CAA TTC AGT ATC CAT AGC TAC TGG GG-3'.

Expression was performed in Escherichia coli XL1 BLUE cells according to Delepelaire and Wandersman (1989). Briefly, cells were grown in LB medium containing $50 \mu \mathrm{g} / \mathrm{ml}$ kanamycin and $50 \mu \mathrm{g} / \mathrm{ml}$ chloramphenicol to an $\mathrm{OD}_{600}$ of 0.6 at $37^{\circ} \mathrm{C}$, then induced with $1 \mathrm{mM}$ isopropyl-beta-D-thiogalactopyrano-side (IPTG) and grown overnight at $37^{\circ} \mathrm{C}$. Secreted proteins were purified by anion exchange chromatography as described previously (Hege and Baumann, 2001a,b). Trigonal-bipyramidal crystals grew in hanging drops at $20^{\circ} \mathrm{C}$ from a solution of $10 \mathrm{mg} /$ $\mathrm{ml}$ protein and a reservoir containing $2 \mathrm{M} \mathrm{NaCl}, 0.1 \mathrm{M} \mathrm{NaH}_{2} \mathrm{PO}_{4}$, $0.1 \mathrm{M} \mathrm{KH}_{2} \mathrm{PO}_{4}, 0.1 \mathrm{M}$ 2-(N-morpholino)ethanesulfonic acid (MES), $\mathrm{pH} 6.5$.

\section{Data collection and structure refinement}

Crystals were cryo-protected by the addition of $25 \%(\mathrm{v} / \mathrm{v})$ glycerol and flash-cooled in a $110 \mathrm{~K}$ nitrogen stream. Data collection in-house employed a Rigaku (Tokyo, Japan) RU300 rotating anode and an RaxisIV image plate detector (Rigaku, Tokyo, Japan). Synchrotron data were collected at the EMBL outstation at Deutsches Elektronensynchrotron (DESY) in Hamburg (Germany) utilizing an MAR165 CCD detector. Data were processed using the HKL2000 package (Otwinowski and Minor, 1997). Interactive model rebuilding was effected with $\mathrm{O}$ (Jones et al., 1991) and COOT (Emsley and Cowtan, 2004). Refinement was initially carried out using CNS (Brunger et al., 1998) and completed by PHENIX using TLS refinement (Adams et al., 2002). Figures were prepared with PYMOL (http://www.pymol.org). Coordinates of the various structures have been deposited in the Protein Data Bank (PDB) under the entry codes 3HB2, $3 \mathrm{HBU}, 3 \mathrm{HBV}$, and 3HDA.

\section{Acknowledgments}

This work has been supported by the Swiss National Science Foundation and by the University of Bern and its supporting foundation. We are grateful to Philippe Delepelaire and Cecile Wandersman, Institute Pasteur, Paris (France) for the plasmids encoding PrtC and the transport machinery. We thank Drs. Manfred S. Weiss and Andrea Schmidt for their support during data collection at the EMBL outstation at DESY, Hamburg (Germany).

\section{References}

Adams, P.D., Grosse-Kunstleve, R.W., Hung, L.W., loerger, T.R., McCoy, A.J., Moriarty, N.W., Read, R.J., Sacchettini, J.C., Sauter, N.K., and Terwilliger, T.C. (2002). PHENIX: building new software for automated crystallographic structure determination. Acta Crystallogr. D Biol. Crystallogr. 58, 19481954.

Baumann, U., Wu, S., Flaherty, K.M., and McKay, D.B. (1993). Three-dimensional structure of the alkaline protease of $P$ seudomonas aeruginosa: a two-domain protein with a calcium binding parallel $\beta$-roll motif. EMBO J. 12, 3357-3364.

Blobel, C.P. (2005). ADAMs: key components in EGFR signalling and development. Nat. Rev. Mol. Cell Biol. 6, 32-43.

Bode, W., Gomis-Ruth, F.X., and Stockler, W. (1993). Astacins, serralysins, snake venom and matrix metalloproteinases exhibit identical zinc-binding environments (HEXXHXXGXXH and Met-turn) and topologies and should be grouped into a common family, the 'metzincins'. FEBS Lett. 331, 134-140.

Bond, J.S. and Beynon, R.J. (1995). The astacin family of metalloendopeptidases. Protein Sci. 4, 1247-1261.

Brunger, A.T., Adams, P.D., Clore, G.M., DeLano, W.L., Gros, P., Grosse-Kunstleve, R.W., Jiang, J.S., Kuszewski, J., Nilges, M., Pannu, N.S., et al. (1998). Crystallography and NMR system: a new software suite for macromolecular structure determination. Acta Crystallogr. D Biol. Crystallogr. 54, 905921.

Butler, G.S., Tam, E.M., and Overall, C.M. (2004). The canonical methionine 392 of matrix metalloproteinase 2 (gelatinase A) is not required for catalytic efficiency or structural integrity: probing the role of the methionine-turn in the metzincin metalloprotease superfamily. J. Biol. Chem. 279, 1561515620.

Delepelaire, P. and Wandersman, C. (1989). Protease secretion by Erwinia chrysanthemi. Proteases $B$ and $C$ are synthesized and secreted as zymogens without a signal peptide. J. Biol. Chem. 264, 9083-9089.

Emsley, P. and Cowtan, K. (2004). Coot: model-building tools for molecular graphics. Acta Crystallogr. D Biol. Crystallogr. 60, $2126-2132$.

Gomis-Ruth, F.X. (2003). Structural aspects of the metzincin clan of metalloendopeptidases. Mol. Biotechnol. 24, 157-202.

Gomis-Ruth, F.X. (2009). Catalytic domain architecture of metzincin metalloproteases. J. Biol. Chem. 284, 15353-15357.

Hege, T. and Baumann, U. (2001a). The conserved methionine residue of the metzincins: a site-directed mutagenesis study. J. Mol. Biol. 314, 181-186. 
Hege, T. and Baumann, U. (2001b). Protease C of Erwinia chrysanthemi: the crystal structure and role of amino acids Y228 and E189. J. Mol. Biol. 314, 187-193.

Jiang, W. and Bond, J.S. (1992). Families of metalloendopeptidases and their relationships. FEBS Lett. 312, 110-114.

Jones, D.H. and Winistorfer, S.C. (1992). Recombinant circle PCR and recombination PCR for site-specific mutagenesis without PCR product purification. Biotechniques 12, 528535.

Jones, T.A., Zou, J.Y., Cowan, S.W., and Kjeldgaard, M. (1991). Improved methods for building protein models in electron density maps and the location of errors in these models. Acta Crystallogr. A 47, 110-119.

Jones, D.T., Taylor, W.R., and Thornton, J.M. (1992). The rapid generation of mutation data matrices from protein sequences. Comput. Appl. Biosci. 8, 275-282.

Lawrence, M.C. and Colman, P.M. (1993). Shape complementarity at protein/protein interfaces. J. Mol. Biol. 234, 946-950.

Lovell, S.C., Davis, I.W., Arendall, W.B. III, de Bakker, P.I., Word, J.M., Prisant, M.G., Richardson, J.S., and Richardson, D.C. (2003). Structure validation by Ca geometry: $\varphi, \psi$ and $\mathrm{Cb}$ deviation. Proteins 50, 437-450.

Maeda, H. and Morihara, K. (1995). Serralysin and related bacterial proteinases. Methods Enzymol. 248, 395-413.

Meier, R., Drepper, T., Svensson, V., Jaeger, K.E., and Baumann, U. (2007). A calcium-gated lid and a large $\beta$-roll sandwich are revealed by the crystal structure of extracellular lipase from Serratia marcescens. J. Biol. Chem. 282, 31477-31483.

Nagase, H., Visse, R., and Murphy, G. (2006). Structure and function of matrix metalloproteinases and TIMPs. Cardiovasc. Res. 69, 562-573.

Otwinowski, Z. and Minor, W. (1997). Processing of X-ray diffraction data collected in oscillation mode. Methods Enzymol. 276, 307-326.
Perez, L., Kerrigan, J.E., Li, X., and Fan, H. (2007). Substitution of methionine 435 with leucine, isoleucine, and serine in tumor necrosis factor $\alpha$ converting enzyme inactivates ectodomain shedding activity. Biochem. Cell Biol. 85, 141-149.

Pieper, M., Betz, M., Budisa, N., Gomis-Ruth, F.X., Bode, W., and Tschesche, H. (1997). Expression, purification, characterization, and X-ray analysis of selenomethionine 215 variant of leukocyte collagenase. J. Protein Chem. 16, 637-650.

Qoronfleh, M.W., Ho, T.F., Brake, P.G., Banks, T.M., Pulvino, T.A., Wahl, R.C., Eshraghi, J., Chowdhury, S.K., Ciccarelli, R.B., and Jones, B.N. (1995). Production of selenomethioninelabeled recombinant human neutrophil collagenase in Escherichia coli. J. Biotechnol. 39, 119-128.

Rawlings, N.D., Morton, F.R., and Barrett, A.J. (2006). MEROPS: the peptidase database. Nucleic Acids Res. 34, D270-D272.

Richardson, J.S. (2003). All-atom contacts: a new approach to structure validation. Methods Biochem. Anal. 44, 305-320.

Stocker, W., Grams, F., Baumann, U., Reinemer, P., Gomis-Ruth, F.X., McKay, D.B., and Bode, W. (1995). The metzincins topological and sequential relations between the astacins, adamalysins, serralysins, and matrixins (collagenases) define a superfamily of zinc-peptidases. Protein Sci. 4, 823-840.

Tourasse, N.J. and Li, W.H. (2000). Selective constraints, amino acid composition, and the rate of protein evolution. Mol. Biol. Evol. 17, 656-664.

Tronrud, D.E., Roderick, S.L., and Matthews, B.W. (1992). Structural basis for the action of thermolysin. Matrix (Suppl) 1 , 107-111.

Walasek, P. and Honek, J.F. (2005). Nonnatural amino acid incorporation into the methionine 214 position of the metzincin Pseudomonas aeruginosa alkaline protease. BMC Biochem. 6, 21.

Received March 15, 2009; accepted May 11, 2009 